\title{
Alignment effects on learning multiple, use-relevant classification systems
}

\author{
CYNTHIA M. SIFONIS \\ Oakland University, Rochester, Michigan \\ and \\ BRIAN H. ROSS \\ University of Illinois at Urbana-Champaign, Urbana, Illinois
}

\begin{abstract}
People often learn multiple classification systems that are relevant to some goal or use. We compared conditions in which subclassification within a category hierarchy was predicted by values on either the same (alignable) or different (nonalignable) dimensions between category hierarchies. The results indicated that learning in alignable conditions occurred in fewer blocks and with fewer errors than did learning in nonalignable conditions. This facilitation was not the result of differences between conditions in the representations learned by the participants, the number of dimensions needed for subclassification (Experiment 1), or the objective complexity of the learning task (Experiment 2). The facilitated learning in the alignable conditions appears to reflect a commitment on the part of the learner to alignment: the belief that the structure relevant to the use of one category system will also be relevant to the use of a comparable system.
\end{abstract}

People often learn about and interact with instances in an unfamiliar domain. In the process, knowledge is built up about the instances, their typical features, and the application of the domain knowledge in a variety of tasks. The result is often an organization of domain knowledge into a category hierarchy that reflects the uses to which the knowledge is applied (Boster \& Johnson, 1989; Medin, Lynch, Coley, \& Atran, 1997; Ross \& Murphy, 1999). In this paper, we will explore the manner in which people learn such hierarchies. Of specific interest are factors affecting how people learn multiple hierarchies simultaneously.

When interacting with category instances, people frequently organize domain knowledge into several related hierarchies. For example, a person learning to train working dogs for a variety of tasks might form distinct subcategories of dogs, such as tracking dogs and service dogs. In working with animals from each category, this person might further subdivide the categories into more specific subcategories. For example, they might subdivide the service dogs

\footnotetext{
Parts of the present manuscript were presented at the annual meeting of the Cognitive Science Society, Vancouver, BC. This study was supported by NSF Grant SBR 97-20304. We thank Gregory L. Murphy, Arthur B. Markman, Bob E. Rehder, and three anonymous reviewers for helpful comments on an earlier version of this manuscript. We also thank Devon Baxter, Angela Castellani, Stacey Delikta, Alex Fedorowich, and Kim Lewis for help in testing participants. Correspondence concerning this article should be addressed to C. M. Sifonis, Department of Psychology, 222 Pryale Hall, Oakland University, Rochester, MI 483094489 (e-mail: sifonis@ oakland.edu).
}

-Accepted by previous editorial team category into vision dogs and hearing dogs. At the same time they might also be subdividing the tracking dogs category into people-tracking dogs and narcotics dogs.

Research has supported the proposal that task-related subdivisions of domain knowledge occur in a wide range of domains. For instance, Ross and Murphy (1999) identified salient organizations of the food domain knowledge of college students resulting from the need to plan and eat several meals a day (e.g., breakfastfoods). Tree experts and fish experts have been shown to possess subdivisions of domain knowledge reflecting goal-directed interactions with members of those domains (e.g., weed trees and game fish; Boster \& Johnson, 1989; Medin et al., 1997). In short, the use of category knowledge frequently has the result that information relevant for that use becomes organized and grouped into goal-relevant subcategories.

Unfortunately, we know little about how people form multiple, goal-relevant category hierarchies. In the research mentioned above, the organization of domain knowledge in well-established domains was examined. Consequently, it does not tell us how people initially learn to classify instances at multiple levels within a domain on the basis of their interactions with category members. The research in which the formation of class-inclusion hierarchies actually has been examined typically has done so for a single superordinate. The focus of these studies often has been on the factors affecting the ease with which category subdivisions are determined (e.g., types of dogs, types of butterfly; Waxman, Lynch, Casey, \& Baer, 1997). One consequence of examining subclassification within a single superordinate is that the focus is on within-category fac- 
tors that affect learning. As a result, we know little about possible between-category factors that could affect the ease with which multiple classification systems are learned.

It is important to investigate how people learn multiple classification hierarchies, because people rarely learn category hierarchies in isolation. Sometimes, we learn categories that bring to mind related categories and learning situations. At other times, we need to learn to distinguish between multiple categories simultaneously and make further goal-driven distinctions within those categories. In the example described previously, the dog trainer learns multiple use-relevant hierarchies. Distinctions at each hierarchical level depend on decisions concerning the types of tasks to which each dog is best suited. If a dog is too aggressive to be a service dog, perhaps it will make a better tracking dog. A dog docile enough to be a service dog might be too small to be an effective vision dog but would make a very good hearing dog.

As the example illustrates, an important part of forming a category hierarchy within a domain is identifying the dimensions important for classification at each level of the hierarchy. In the preceding example, values on the temperament dimension (i.e., aggression) allow the trainer to distinguish between service dogs and tracking dogs. For service dogs, values on the dimension of size determine the most effective use of the dog and allow the trainer to make distinctions between types of service dogs (vision dogs vs. hearing dogs).

Identifying the dimensions on which divisions within a hierarchy can be made is facilitated when those divisions can be made on the basis of contrastive information (Billman, 1996; Waxman et al., 1997). Contrastive information refers to an aspect of category structure in which the same dimensions matter across the category. When values on the same dimension allow a person to distinguish between categories at the same level of a hierarchy, learning is facilitated. This is true whether one is learning to distinguish between basic level categories (Kaplan, 2000; Lassaline \& Murphy, 1998) or learning to make subordinate level distinctions within a category (Billman, 1996; Waxman et al., 1997). It has been assumed that this facilitation reflects assumptions made on the part of the learner about the structure of the categories being learned. However, as will be discussed shortly, such facilitation may be a by-product of the designs used to study the phenomenon. Given the evidence that similarities in category structure facilitate classification learning at several hierarchical levels, it is extremely important to investigate the source of this facilitation.

The present study extends the work reported in Sifonis and Ross (1999) demonstrating that between-category similarities in the dimensions important for the use of category knowledge facilitate the learning of multiple classification systems. The source of this facilitation was explored in two experiments. In the first experiment, we examined whether facilitation is due to the number of dimensions needing attention in order to make subordinate level category distinctions. In the second experiment, we examined whether the facilitation is a product of the psychological complexity of the learning task. ${ }^{1}$
In the present work, we chose the domain of bank loan applications for two reasons. It is a complex domain that is similar to some real-world category learning, and it also allows the learning of multiple, goal-driven category distinctions. When presented with a member of the domain (a loan application form), participants decided whether or not the applicant would receive the loan. This initial decision established the superordinate categories within which the participants learned to make further goal-relevant category distinctions. After the loan application was categorized into one of the two superordinate categories, the participants decided what type of financial advice to give the applicant and/or what consultant to assign to the applicant. The participants learned two hierarchies, each containing subordinate categories derived from the uses to which category knowledge was applied (deciding financial advice and/or consultant).

\section{Structural Alignment}

Some of the work in which the effects of contrastive information on classification learning were examined has used the concept of structural alignment to predict and explain classification performance (Goldstone, 1994; Kaplan, 2000; Lassaline \& Murphy, 1998). Structural alignment theory assumes that category representations encode such information as the dimensions associated with the category, the features typically instantiated by those dimensions, and the relationships between dimensions. Betweencategory comparisons involve aligning these representations to find the most structurally consistent match between them (Gentner, 1983). The alignment process highlights structural consistencies and inconsistencies between representations. Structural consistencies known as commonalities involve between-category matches on both dimensions and features. For example, if both loan-suitable and loan-unsuitable companies advertise in magazines, this would be a commonality in their representations. There is a between-category match on dimension (method of advertising) and feature (magazine). Differences in representational structure can be either alignable or nonalignable. Alignable differences involve a match on dimension but a mismatch on features. Loan-suitable and loan-unsuitable companies would have alignable differences if the loansuitable company advertised by television, whereas the loan-unsuitable company advertised by newspaper. Nonalignable differences, on the other hand, are independent of the common structure. Differences of this type involve dimensions that have no corresponding dimension for the other entity. For instance, perhaps included on the loan application of a grocery company are dimensions representing shipping costs and distribution. These dimensions would not be applicable to companies specializing in Web development and would not be included on their loan applications.

\section{Alignable Differences and Learning Use-Relevant Classification Systems}

The distinction between alignable and nonalignable differences has proven useful for predicting classification 
learning at both basic (Kaplan, 2000, Experiments 2 and 3; Lassaline \& Murphy, 1998) and subordinate (Waxman et al., 1997) levels in a hierarchy. It also predicts the ease with which multiple classification systems are learned (Billman, 1996; Sifonis \& Ross, 1999). Of particular interest to the present study is the demonstration that betweencategory consistencies in the use of category knowledge affect the formation of use-relevant subordinate cate-

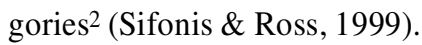

As in the present study, Sifonis and Ross (1999) had participants make several classification distinctions based on information in bank loan application forms. The participants initially classified the loan applications into the superordinate categories of applications that received loans (loan category) and those that did not receive loans (no-loan category). The participants then had to make financial advice decisions based on superordinate category membership (loan/no-loan) and information found in the applications. The between-category alignability of the dimensions necessary for predicting financial advice was varied between conditions. In the alignable-use condition, the same dimension (i.e., type of sales) predicted financial advice for both superordinate categories of loan applications. In the nonalignable-use condition, the dimensions predicting financial advice differed between superordinates (i.e., type of sales for applications receiving a loan and method of advertising for applications that did not receive a loan). It was found that when the relationship between a dimension and its use was consistent between categories, learning the classification hierarchies was facilitated.

Sifonis and Ross's (1999) study demonstrated that between-category consistencies in the dimensions predictive of subclassification within a hierarchy affect the ease with which multiple classification hierarchies are learned. It also avoided an important confound of previous studies in which alignment effects on classification were examined. Specifically, in Sifonis and Ross's study, there were no differences between conditions in the category representations learned by participants.

Typically, experiments in which alignment effects on classification learning have been examined have manipulated alignability in terms of whether contrastive categories possess alignable or nonalignabledimensions predictive of category membership (Kaplan, 2000, Experiments 2 and 3; Wisniewski \& Markman, 1997, Experiments 1 and 2). By necessity, the contrast category representations and the exemplars experienced by participants during learning differed between conditions. By examining how people learn use-relevant subclassifications within multiple hierarchies, it becomes possible to manipulate the alignability of the dimensions important for category use between conditions while holding the alignability of the category representations constant.

In Sifonis and Ross's (1999) study, the category representations learned by participants were identical between conditions. Both categories of loan applications possessed the same dimensions in both the alignable-use and the nonalignable-useconditions. The participants in both conditions experienced exactly the same category exemplars.
As a result, the facilitated learning seen in the alignableuse condition of that study could not be attributed to differences between conditions in the category representations learned by the participants. However, it is still possible that the facilitation arose from a source other than the alignability of the dimensions predictive of subclassification within each hierarchy.

To date, all the studies in which multiple classification system learning has been examined have ensured that the number of features needed to make the classification and subclassification decisions were equated across conditions. However, the same cannot be said for the number of dimensions associated with those features (Billman, 1996; Sifonis \& Ross, 1999). Consequently, it is possible that the alignability effects exhibited in previous studies resulted from differences between conditions in the number of dimensions needed to make classification decisions. By definition, a single classification decision made on the basis of alignable differences between categories requires attention to a single dimension (e.g., type of sales: retaill type of sales: wholesale). In contrast, decisions made on the basis of nonalignable differences between categories require attention to several dimensions (type of sales: retaill method of advertising: magazine). In order to determine the independent contribution of alignability to the learning of multiple classification systems, it is necessary to separate alignability from the number of dimensions necessary for making category distinctions.

\section{EXPERIMENT 1}

Experiment 1 eliminated the confound between alignability and the number of attended dimensions by requiring that participants learn to make two use-relevant distinctions within each superordinate category. As in Sifonis and Ross (1999), the participants reviewed bank loan application forms and classified the applications at both the superordinate and the subordinate levels. However, in the present experiment, the participants in both the alignable-use and the nonalignable-use conditions were required to pay attention to two dimensions in order to classify at the subordinate level. The alignability of these dimensions was manipulated by varying the uses predicted by the dimensions in each of the two superordinate categories. In the alignable-use condition, the same dimension was used to predict the same use in both superordinate categories of applications (i.e., Dimension 1 predicted Use 1 and Dimension 2 predicted Use 2 for both the loan and the no-loan categories). In the nonalignable-use condition, the uses predicted by the dimensions changed for each superordinate (i.e., Use 1 was predicted by Dimension 1 and Use 2 by Dimension 2 for the loan category, and Use 1 was predicted by Dimension 2 and Use 2 by Dimension 1 for the no-loan category). Consequently, and in contrast to previous studies (Billman, 1996), alignabilityreferred to the alignability of the use-relevant dimensions between superordinates, rather than between the categories sharing a superordinate, in the present study.

To the extent that between-category regularities in the use of category knowledge are a factor in learning multi- 


\begin{tabular}{|c|c|}
\hline $\begin{array}{l}\text { Application for Business } \\
\text { Expansion Loan }\end{array}$ & $\begin{array}{l}\text { Guarantor Identification } \\
\text { First National } \\
\text { City Bank }\end{array}$ \\
\hline $\begin{array}{l}\text { OMB No. 1840-0717 Form Approved Exp. Date } \\
\text { 03/31/99 }\end{array}$ & \\
\hline $\begin{array}{l}\text { Warning: Any person who knowingly makes a faise } \\
\text { statement or missepresentation on this form is to penalties } \\
\text { which may include fines or imprisonment under the } \\
\text { United States Criminal Code and } 20 \text { U.S.C. } 1097\end{array}$ & t \\
\hline $\begin{array}{l}\text { Borrower Section } \\
\text { carefully }\end{array}$ & atly or type. Read the instructions \\
\hline 1. Company Name & 2. Company Address \\
\hline $\begin{array}{l}\text { 3. Industry } \\
\text { Drug }\end{array}$ & $\begin{array}{l}\text { 4. Type of Sales } \\
\text { Retail }\end{array}$ \\
\hline $\begin{array}{l}\text { 5. International Market } \\
\text { Europe }\end{array}$ & $\begin{array}{l}\text { 6. Method of Advertising } \\
\text { Radio }\end{array}$ \\
\hline 7. Loan Period (Month/Year) & 8. Loan Assistance Requested \\
\hline $9 / 98-9 / 03$ & $\$ 10,000,000.00$ \\
\hline \multicolumn{2}{|l|}{ Promissory Note } \\
\hline \multicolumn{2}{|c|}{$\begin{array}{l}\text { Promise to Pay: I promise to pay to the lender, or a subsequent holder of this Promissory Note, all sums } \\
\text { dispersed (hear after "loan" or "loans") under the terms of this note, plus interest and other fees which } \\
\text { may become due as provided by this Note. If I fail to make payments on this Note when due, I will also } \\
\text { pay reasonable collection costs, including attorney's fees, court costs, and collection fees. I understand } \\
\text { that I may cancel or reduce the size of any loan by refusing to accept any disbursement that is issued. }\end{array}$} \\
\hline \multicolumn{2}{|l|}{ THIS IS A LOAN(S) THAT MUST BE REPAID } \\
\hline \multicolumn{2}{|c|}{ 9. Borrower's Signature ___ _ _ _ Today's Date (Month/Day/Year) } \\
\hline Bank Section & leted by an authorized bank official \\
\hline 10. Lender Name & 11. Lender Code \\
\hline First National City Bank & $100 \times X 1 X 2$ \\
\hline $\begin{array}{l}\text { 12. Telephone Number } \\
\text { (217) } 333-2012\end{array}$ & $\begin{array}{l}\text { 13. Amount(s) Approved } \\
\text { \$ }\end{array}$ \\
\hline
\end{tabular}

Figure 1. Loan application form.

ple classification hierarchies, the alignability of userelevant dimensions should affect how quickly these hierarchies are learned. When alignable dimensions predict subclassification in both hierarchies, we predict that learning those hierarchies will proceed more quickly and with fewer errors than when the dimensions are nonalignable.If the alignability effects seen in previous experiments were due simply to the number of dimensions needing attention in order to learn how to use the category, there should be no effects of alignability in the present experiment.

\section{Method}

Participants. The participants consisted of 48 University of Illinois students, who participated for experimental credit or pay. The sessions lasted from $1 \mathrm{~h}$ to $1 \mathrm{~h}$ and $50 \mathrm{~min}$.
Materials and Design. The materials were constructed to look like bank loan application forms (see Figure 1). Included on the forms were four, four-featured dimensions: Sales (wholesale, retail, mail order, or Internet), advertising (television, radio, magazine, or newspaper), product (electronics, drugs, food, or home furnishings), and international market (Asia, South America, Europe, or Australia).

Thirty-two loan applications were constructed, 16 in each category. Table 1 shows the abstract structure for the two categories of loan applications for each condition. Each dimension on the application (D1-D4) contained one of four possible features, denoted in the columns as values ranging from one to four. The features of two dimensions (D1 and D2) were perfectly predictive of superordinate category membership (superordinate-relevant dimensions).

Both of the superordinate-relevant dimensions were also predictive of the use of the category (use-relevant dimensions). One of the two use-relevant dimensions (indicated in bold type in Table 1) was predictive of the financial advice given to the applicant. The other 
Table 1

Experiment 1: Design

\begin{tabular}{|c|c|c|c|c|c|c|c|c|c|c|c|c|}
\hline \multirow[b]{2}{*}{ Item } & \multicolumn{6}{|c|}{ Loan Category Exemplars } & \multicolumn{6}{|c|}{ No-Loan Category Exemplars } \\
\hline & D1 & D2 & D3 & $\mathrm{D} 4$ & Use1 & Use2 & D1 & D2 & D3 & D4 & Use1 & Use2 \\
\hline \multicolumn{13}{|c|}{ Exemplars for the Alignable-Use Condition } \\
\hline 1 & 1 & 1 & 1 & 4 & 1 & 1 & 3 & 3 & 1 & 4 & 3 & 3 \\
\hline 2 & 1 & 2 & 2 & 3 & 1 & 2 & 3 & 4 & 2 & 3 & 3 & 4 \\
\hline 3 & 1 & 1 & 3 & 2 & 1 & 1 & 3 & 3 & 3 & 2 & 3 & 3 \\
\hline 4 & 1 & 2 & 4 & 1 & 1 & 2 & 3 & 4 & 4 & 1 & 3 & 4 \\
\hline 5 & 1 & 1 & 2 & 3 & 1 & 1 & 3 & 3 & 2 & 3 & 3 & 3 \\
\hline 6 & 1 & 2 & 3 & 2 & 1 & 2 & 3 & 4 & 3 & 2 & 3 & 4 \\
\hline 7 & 1 & 1 & 4 & 1 & 1 & 1 & 3 & 3 & 4 & 1 & 3 & 3 \\
\hline 8 & 1 & 2 & 1 & 4 & 1 & 2 & 3 & 4 & 1 & 4 & 3 & 4 \\
\hline 9 & 2 & 1 & 3 & 2 & 2 & 1 & 4 & 3 & 3 & 2 & 4 & 3 \\
\hline 10 & 2 & 2 & 4 & 1 & 2 & 2 & 4 & 4 & 4 & 1 & 4 & 4 \\
\hline 11 & 2 & 1 & 1 & 4 & 2 & 1 & 4 & 3 & 1 & 4 & 4 & 3 \\
\hline 12 & 2 & 2 & 2 & 3 & 2 & 2 & 4 & 4 & 2 & 3 & 4 & 4 \\
\hline 13 & 2 & 1 & 4 & 1 & 2 & 1 & 4 & 3 & 4 & 1 & 4 & 3 \\
\hline 14 & 2 & 2 & 1 & 4 & 2 & 2 & 4 & 4 & 1 & 4 & 4 & 4 \\
\hline 15 & 2 & 1 & 2 & 3 & 2 & 1 & 4 & 3 & 2 & 3 & 4 & 3 \\
\hline 16 & 2 & 2 & 3 & 2 & 2 & 2 & 4 & 4 & 3 & 2 & 4 & 4 \\
\hline \multicolumn{13}{|c|}{ Exemplars for the Nonalignable-Use Condition } \\
\hline & D1 & D2 & D3 & D4 & Use1 & Use2 & D1 & D2 & D3 & D4 & Use1 & Use 2 \\
\hline 1 & 1 & 1 & 1 & 4 & 1 & 1 & 3 & 3 & 1 & 4 & 3 & 3 \\
\hline 2 & 1 & 2 & 2 & 3 & 1 & 2 & 3 & 4 & 2 & 3 & 4 & 3 \\
\hline 3 & 1 & 1 & 3 & 2 & 1 & 1 & 3 & 3 & 3 & 2 & 3 & 3 \\
\hline 4 & 1 & 2 & 4 & 1 & 1 & 2 & 3 & 4 & 4 & 1 & 4 & 3 \\
\hline 5 & 1 & 1 & 2 & 3 & 1 & 1 & 3 & 3 & 2 & 3 & 3 & 3 \\
\hline 6 & 1 & 2 & 3 & 2 & 1 & 2 & 3 & 4 & 3 & 2 & 4 & 3 \\
\hline 7 & 1 & 1 & 4 & 1 & 1 & 1 & 3 & 3 & 4 & 1 & 3 & 3 \\
\hline 8 & 1 & 2 & 1 & 4 & 1 & 2 & 3 & 4 & 1 & 4 & 4 & 3 \\
\hline 9 & 2 & 1 & 3 & 2 & 2 & 1 & 4 & 3 & 3 & 2 & 3 & 4 \\
\hline 10 & 2 & 2 & 4 & 1 & 2 & 2 & 4 & 4 & 4 & 1 & 4 & 4 \\
\hline 11 & 2 & 1 & 1 & 4 & 2 & 1 & 4 & 3 & 1 & 4 & 3 & 4 \\
\hline 12 & 2 & 2 & 2 & 3 & 2 & 2 & 4 & 4 & 2 & 3 & 4 & 4 \\
\hline 13 & 2 & 1 & 4 & 1 & 2 & 1 & 4 & 3 & 4 & 1 & 3 & 4 \\
\hline 14 & 2 & 2 & 1 & 4 & 2 & 2 & 4 & 4 & 1 & 4 & 4 & 4 \\
\hline 15 & 2 & 1 & 2 & 3 & 2 & 1 & 4 & 3 & 2 & 3 & 3 & 4 \\
\hline 16 & 2 & 2 & 3 & 2 & 2 & 2 & 4 & 4 & 3 & 2 & 4 & 4 \\
\hline
\end{tabular}

Note-D1-D4 indicate exemplar dimensions and their features. Feature values of 1 or 2 for Dimensions D1 or D2 are predictive of the loan category. Values of 3 or 4 for Dimensions D1 or D2 are predictive of the no-loan category. Use 1 indicates the financial advice decision. Use 2 indicates the consultant decision. The dimensions in bold correspond to and predict the financial advice in bold. The dimensions in italics correspond to and predict the consultant advice in italics.

dimension (indicated in italics in Table 1) was predictive of the consultant decision. The two remaining dimensions did not predict membership in either the superordinate or the subordinate level categories.

Take, for example, the alignable-use condition in Table 1. Values on both the sales dimension (D1) and the advertising dimension (D2) predicted membership in the superordinate category. If the feature of sales was wholesale (indicated by a 1 ) or retail (indicated by a 2 ) or the feature of advertising was television (indicated by a 1) or radio (indicated by a 2), the applicant received the loan. If the feature of sales was mail order (indicated by a 3 ) or Internet (indicated by a 4) or the feature of advertising was magazine (indicated by a 3 ) or newspaper (indicated by a 4), the applicant did not receive the loan.

In this example, sales and advertising were also use-relevant dimensions, predicting subclassification within each superordinate. In the loan category, if the value of sales was wholesale, the financial advice given was product development (indicated by a 1 in the Use 1 column). If it was retail, the financial advice was expand line of merchandise (as indicated by a 2 in the Use1 column). Similarly, if the value of advertising was television, the consultant was Ward (indicated by a 1 in the Use 2 column). If the value of advertising was radio, the consultant was Smith (indicated by a 2 in the Use 2 col- umn). In the no-loan category, sales (mail order or Internet) also predicted financial advice (sell some stock or focus on specialized market), and advertising (magazine or newspaper) predicted the consultant decision (Jones or Brown).

As can be seen in Table 1, the exemplars for the loan and the noloan categories were identical in both the alignable-use and the nonalignable-use conditions. The superordinate-relev ant and the userelevant dimensions were also identical. The difference between conditions was in the alignability of dimensions predictive of each use of the category. In the alignable-use condition, one superordinaterelevant dimension predicted financial advice for both categories, and the other predicted the consultant for both categories. In the nonalignable-use condition, the dimension predictive of financial advice for one category predicted the consultant for the contrast category. For example, for the nonalignable-use condition, the loan category might be as above, but the no-loan category would switch dimensions predictive of the uses, with advertising predicting financial advice and sales predicting consultant.

To ensure that any learning differences between conditions was not due to the particular dimensions and their features, the use-relevant dimensions predictive of each use were counterbalanced over participants. 
Procedure. The participants were told that the experiment examined how people learn to classify items and then use that information to make decisions related to the category. They were instructed to imagine that they were junior bank loan executives learning how to classify loan applications into those that would receive business expansion loans and those that would not. Classification would be based on the information in the company's loan application form. After classifying the loan application, they would then use the information in the application to determine what financial advice to give the applicant. For companies that received a loan, the advice referred to how the loan money was to be spent (expand the line of merchandise or product development). For companies that did not receive a loan, the advice referred to how the company could generate revenue (sell some stock or focus on specialized market). Following the financial advice decision, they would then use the information in the application to determine which consultant would further process the loan. As with financial advice, the consultant decision consisted of four choices (two for each superordinate category) predicted by the variables in the loan application. Two of the choices were appropriate for applications receiving a loan (Smith or Ward), and two choices were appropriate for applications that did not receive a loan (Jones or Brown).

A sheet with the loan categories (loan/no-loan) and the corresponding financial advice and consultant decisions was visible throughout the experiment. The participants were given an example of the loan application form with Xs replacing the features for the four dimensions. Their attention was then directed to those dimensions, and they were told that they would use the information contained in those dimensions to make the loan, financial advice, and consultant decisions.

On each study trial, the participants received a loan application form containing values on the four dimensions and made three responses. First, they classified the item (received a loan/did not receive a loan). Second, using the correct category, the participants then responded with one of the financial advice decisions. Finally, the participants responded with one of the consultant decisions. Following each response, the experimenter provided feedback about the correctness of each classification. The participants were given as much time as they wished to study the application before the next exemplar was presented.

Each block consisted of the random presentation of 16 applications ( 8 loan and 8 no-loan). Consequently, it took 2 complete blocks for the participant to see all 32 loan applications. All the participants engaged in learning trials until they made no more than two mistakes on a block (out of 48 responses) or until they had completed 12 blocks.

For the analysis of blocks taken to learn both the superordinate ${ }^{3}$ and the subordinate category distinctions, the participants who had not learned the loan/no-loan distinction, the financial advice, and/or the consultant decisions were recorded as having learned them by the 13th block. This was done as a conservative estimate of the next block at which they could have reached criterion had they been allowed to continue. To examine the effects of alignability on subclassification learning, we averaged the number of blocks it took to learn each use-relevant subordinate category distinction, to produce a combined blocks-to-learn-use score. We did the same with the number of errors before both uses were learned to produce an errorsto-learn-use score.

\section{Results and Discussion}

If between-category alignability facilitates learning subordinate level distinctions, learning should have been facilitated in the alignable-use condition, because the same dimensions predicted subclassification in both hierarchies. Earlier research had shown an advantage for this condition when it was confounded with fewer attended dimensions. Would we still see facilitated learning in the alignable-use condition when the conditions were equated on the number of attended dimensions?

The distributions of scores were positively skewed, so log transformations were performed. There was also significant variability introduced within each condition by the counterbalancing manipulation. To remove this source of variance for between-condition analyses, the log-transformed scores in the two counterbalancings within each condition were adjusted to have the same mean as the overall condition mean. This adjustment reduced the variability within each condition without affecting the condition means. Backtransformed means and standard deviations will be reported.

When the dimensions predictive of use-relevant subclassifications within a hierarchy were alignable between hierarchies, learning was facilitated. As can be seen in Table 2, the participants in the alignable-use conditionlearned the use-relevant subcategories for each hierarchy in fewer blocks and with fewer errors than did participants in the nonalignable-usecondition $[t(46)=2.67, p<.01 ; t(46)=$ $2.12, p<.05]$. So, even when the number of attended dimensions was equated, the alignable-use condition showed an advantage in learning.

This learning advantage also held for each individual subclassification. The participants in the alignable-use condition learned the financial advice decisions in fewer blocks (5.9 vs. 8.7) and made fewer errors (28.3 vs. 44.8) than did those in the nonalignable-use condition $[t(46)=$ $2.77, p<.01 ; t(46)=2.38, p<.05]$. The same was true when the consultant decisions were learned [alignable, blocks $=5.2$, errors $=26.8$; nonalignable, blocks $=8.0$, errors $=42.5 ; t(46)=2.54, p<.05 ; t(46)=1.90, p=.06]{ }^{4}$

To summarize, between-category consistencies in the dimensions predictive of subclassification within a category facilitated the learning of multiple, use-relevant clas-

Table 2

Experiment 1: The Effects of Alignable Uses on Learning Multiple Classification Systems

\begin{tabular}{|c|c|c|c|c|c|c|c|c|}
\hline & \multicolumn{2}{|c|}{ Use Blocks } & \multicolumn{2}{|c|}{ Use Errors } & \multicolumn{2}{|c|}{ Loan Blocks } & \multicolumn{2}{|c|}{ Loan Errors } \\
\hline & Align & Nonalign & Align & Nonalign & Align & Nonalign & Align & Nonalign \\
\hline $\bar{M}$ & 5.7 & 8.3 & 29.5 & 45.0 & 3.1 & 4.2 & 8.3 & 11.2 \\
\hline$S D$ & 3.1 & 3.7 & 21.8 & 29.9 & 2.4 & 3.0 & 10.4 & 14.7 \\
\hline
\end{tabular}

Note-Align indicates the scores for the alignable-use condition. Nonalign indicates the scores for the nonalignable-use condition. Use Blocks/Errors indicate the mean number of blocks/errors to learn both the financial advice and consultant decisions (averaged across both uses). Loan Blocks/Errors indicate the mean number of blocks/errors to learn the loan/no-loan decision. 
sification systems. Even when the number of dimensions needed for subclassification was held constant between conditions, we still saw alignability effects on learning. When the dimensions important for subclassification were alignable between superordinates, learning was enhanced.

Experiment 1 controlled variables left to vary in other studies in which the effect of alignment on classification learning was examined. As in Sifonis and Ross (1999), the present experiment eliminated differences between conditions in the category representations. Most important, it separated alignability from the number of dimensions important for making distinctions within a category. Demonstrating alignability effects while controlling for differences between conditions in the category representations and the number of attended dimensions suggests that such effects do not appear to be solely an artifact of the experimental designs used to study them.

Even though the alignability effects observed in Experiment 1 can no longer be attributed to the number of attended dimensions, it is possible that such effects arose from something other than the between-category alignability of the dimensions predictive of subclassification. An alternative explanation is that the alignable-use condition required the learning of functions that were less complex than those in the nonalignable-use condition.5 There are different ways in which one can think about the complexity of the functions in Experiment 1. One possibility is to consider it at the level of the mapping between features (e.g., sales: wholesale) and the particular use values (e.g., financial advice: product development). The number of mappings between feature and use values was the same for each condition (eight). In addition, the difficulty of classification at the superordinate level (loan/no-loan) was exactly the same for the two conditions. So, if people were simply learning the mappings between features and use values, there was no difference in complexity.

Another possibility, however, is to consider complexity in terms of how dimensions, not features, related to uses. In the alignable-use condition, the association between a dimension and its use remained the same in both category systems, whereas in the nonalignable-use condition, the association between dimension and use changed as a function of superordinate category membership. Thus, by this view of complexity, the alignable-use condition was less complex. Even though the number of dimensions needed for category use was held constant between conditions, the manner in which those dimensions were used between category systems varied by condition. The complexity argument maintains that this difference required that something additional be learned in the nonalignable-usecondition, so learning was more difficult.

It is difficult to separate this type of complexity argument from the alignability argument, because the difference in the relation between the dimensions and the uses is both a complexity and an alignability difference. The important point about the complexity argument is that it does not view any particular complexity as critical, just that overall complexity will determine learning. In contrast, the alignability account claims that overall complexity is not the primary determinant of ease of learning but, rather, that one type of complexity, alignability, is especially important. In other words, the argument is one of how complexity relates to psychological complexity. The complexity view is that psychologicalcomplexity (e.g., as measured by difficulty in learning) is a function of overall objective complexity. The alignability view is that some types of complexity - in particular, alignability - are especially influential. We do not dispute that, keeping everything else constant, variations in alignability will lead to differences in complexity. We believe that between-category consistencies in how dimensions are used are a component of what it means for categories or classification systems to be alignable. Our claim is that the large differences in learning in Experiment 1 were due to this relatively subtle difference in a particular complexity, alignability. Despite the fact that alignability is usually confounded with overall complexity, this does not mean that the two views are indistinguishable. The purpose of Experiment 2 was to investigate whether overall complexity is sufficient for understanding the learning facilitation in the alignable-use condition.

\section{EXPERIMENT 2}

Experiment 2 compared learning in a complex alignable condition to learning in a less complex, nonalignablecondition. The nonalignablecondition was made less complex than the alignable condition by requiring only half as many category use decisions, as compared with the alignable condition. The participants in the alignable-use condition were treated exactly as in Experiment 1 (see the top half of Table 3). They had to learn the loan/no-loan superordinate category distinctions and both use-relevant subordinate level distinctions. The participants in the nonalignableuse condition learned only a subset of what was required in Experiment 1 (see the bottom half of Table 3). They had to learn the same loan/no-loan superordinate category distinctions as the participants in the alignable-use condition. However, they had to learn only one of the two use-relevant subordinate level distinctions (e.g., financial advice, but not consultant). Because subclassification in the nonalignableuse condition was a subset of subclassification in the alignable-use condition, the complexity was reduced relative to the alignable-use condition. The participants had fewer distinctionsto learn at one time and four fewer featureto-use mappings to establish. Note that the conditions remained equivalent in terms of the category representations and the number of dimensions needed to make userelevant distinctions within each category. The conditions differed only in the alignability of the dimensions predictive of category use and the complexity of the learning task.

Although one can argue about how to equate complexity of alignability with the complexity of feature-value mappings, our approach was to make the nonalignableuse condition less complex in terms of the feature-value 
Table 3

Experiment 2: Design

\begin{tabular}{|c|c|c|c|c|c|c|c|c|c|c|c|c|}
\hline \multirow[b]{2}{*}{ Item } & \multicolumn{6}{|c|}{ Loan Category Exemplars } & \multicolumn{6}{|c|}{ No-Loan Category Exemplars } \\
\hline & D1 & $D 2$ & D3 & D4 & Use1 & Use2 & D1 & $D 2$ & D3 & D4 & Use1 & Use2 \\
\hline \multicolumn{13}{|c|}{ Exemplars for the Alignable-Use Condition } \\
\hline 1 & 1 & 1 & 1 & 4 & 1 & 1 & 3 & 3 & 1 & 4 & 3 & 3 \\
\hline 2 & 1 & 2 & 2 & 3 & 1 & 2 & 3 & 4 & 2 & 3 & 3 & 4 \\
\hline 3 & 1 & 1 & 3 & 2 & 1 & 1 & 3 & 3 & 3 & 2 & 3 & 3 \\
\hline 4 & 1 & 2 & 4 & 1 & 1 & 2 & 3 & 4 & 4 & 1 & 3 & 4 \\
\hline 5 & 1 & 1 & 2 & 3 & 1 & 1 & 3 & 3 & 2 & 3 & 3 & 3 \\
\hline 6 & 1 & 2 & 3 & 2 & 1 & 2 & 3 & 4 & 3 & 2 & 3 & 4 \\
\hline 7 & 1 & 1 & 4 & 1 & 1 & 1 & 3 & 3 & 4 & 1 & 3 & 3 \\
\hline 8 & 1 & 2 & 1 & 4 & 1 & 2 & 3 & 4 & 1 & 4 & 3 & 4 \\
\hline 9 & 2 & 1 & 3 & 2 & 2 & 1 & 4 & 3 & 3 & 2 & 4 & 3 \\
\hline 10 & 2 & 2 & 4 & 1 & 2 & 2 & 4 & 4 & 4 & 1 & 4 & 4 \\
\hline 11 & 2 & 1 & 1 & 4 & 2 & 1 & 4 & 3 & 1 & 4 & 4 & 3 \\
\hline 12 & 2 & 2 & 2 & 3 & 2 & 2 & 4 & 4 & 2 & 3 & 4 & 4 \\
\hline 13 & 2 & 1 & 4 & 1 & 2 & 1 & 4 & 3 & 4 & 1 & 4 & 3 \\
\hline 14 & 2 & 2 & 1 & 4 & 2 & 2 & 4 & 4 & 1 & 4 & 4 & 4 \\
\hline 15 & 2 & 1 & 2 & 3 & 2 & 1 & 4 & 3 & 2 & 3 & 4 & 3 \\
\hline 16 & 2 & 2 & 3 & 2 & 2 & 2 & 4 & 4 & 3 & 2 & 4 & 4 \\
\hline
\end{tabular}

Exemplars for the Nonalignable-Use Condition

\begin{tabular}{|c|c|c|c|c|c|c|c|c|c|c|}
\hline & D1 & $\mathrm{D} 2$ & D3 & D4 & Use & D1 & D2 & D3 & D4 & Use \\
\hline 1 & 1 & 1 & 1 & 4 & 1 & 3 & 3 & 1 & 4 & 3 \\
\hline 2 & 1 & 2 & 2 & 3 & 1 & 3 & 4 & 2 & 3 & 4 \\
\hline 3 & 1 & 1 & 3 & 2 & 1 & 3 & 3 & 3 & 2 & 3 \\
\hline 4 & 1 & 2 & 4 & 1 & 1 & 3 & 4 & 4 & 1 & 4 \\
\hline 5 & 1 & 1 & 2 & 3 & 1 & 3 & 3 & 2 & 3 & 3 \\
\hline 6 & 1 & 2 & 3 & 2 & 1 & 3 & 4 & 3 & 2 & 4 \\
\hline 7 & 1 & 1 & 4 & 1 & 1 & 3 & 3 & 4 & 1 & 3 \\
\hline 8 & 1 & 2 & 1 & 4 & 1 & 3 & 4 & 1 & 4 & 4 \\
\hline 9 & 2 & 1 & 3 & 2 & 2 & 4 & 3 & 3 & 2 & 3 \\
\hline 10 & 2 & 2 & 4 & 1 & 2 & 4 & 4 & 4 & 1 & 4 \\
\hline 11 & 2 & 1 & 1 & 4 & 2 & 4 & 3 & 1 & 4 & 3 \\
\hline 12 & 2 & 2 & 2 & 3 & 2 & 4 & 4 & 2 & 3 & 4 \\
\hline 13 & 2 & 1 & 4 & 1 & 2 & 4 & 3 & 4 & 1 & 3 \\
\hline 14 & 2 & 2 & 1 & 4 & 2 & 4 & 4 & 1 & 4 & 4 \\
\hline 15 & 2 & 1 & 2 & 3 & 2 & 4 & 3 & 2 & 3 & 3 \\
\hline 16 & 2 & 2 & 3 & 2 & 2 & 4 & 4 & 3 & 2 & 4 \\
\hline
\end{tabular}

Note-D1-D4 indicate exemplar dimensions and their features. Feature values of 1 or 2 for Dimension D1 or D2 are predictive of the loan category. Values of 3 or 4 for Dimensions D1 or D2 are predictive of the no-loan category. For the alignableuse condition, Use1 indicates the financial advice decision, and Use 2 indicates the consultant decision. For the nonalignableuse condition, Use indicates either the financial advice or the consultant decision. The dimensions in bold correspond to and predict the financial advice in bold. For the alignable-use condition, the dimensions in italics correspond to and predict the consultant advice in italics.

mappings (four instead of eight), as can be seen from a comparison of the two conditionsin Table 3. If overall complexity was the reason behind the results of Experiment 1, one would expect facilitated learning in the less complex, nonalignable condition of Experiment 2. The participants should learn to make use-relevant category distinctions in fewer blocks and with fewer errors in the nonalignableuse condition than in the alignable-use condition. If, however, differences between conditions in alignability produced the results in Experiment 1, even this large reduction of objective complexity in the nonalignable-usecondition, relative to that in the alignable-use condition, might not be enough to overcome the alignability differences. Thus, even with only one use to learn (and far fewer mappings), the participants in the nonalignable-use condition might not learn the classification systems in fewer blocks or make fewer errors than the participants in the alignableuse condition.

\section{Method}

Participants. The participants consisted of 64 Oakland University students, who participated as volunteers or for experimental credit. The data from 8 participants were excluded because they failed to learn the classification distinction (loan/no-loan) within 16 blocks or because time ran out before they were able to finish all the learning blocks. ${ }^{6}$ The data from the remaining 56 participants were analyzed. The sessions lasted from $1 \mathrm{~h}$ to $1 \mathrm{~h}$ and $50 \mathrm{~min}$.

Materials and Design. The study materials were the same as those in Experiment 1. The design was similar to that used in Experiment 1 (see Table 3). Complexity was manipulated in terms of the number of category use decisions the participants needed to learn in each condition. In the alignable-use condition, the participants had to learn both the financial advice and the consultant decisions. In design, the alignable-use condition of the present experiment was 
Table 4

Experiment 2: The Effects of Complexity on Learning Multiple Classification Systems

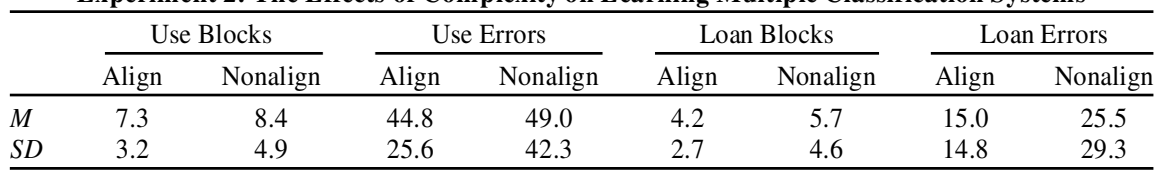

Note-Align indicates the scores for the alignable-use condition. Nonalign indicates the scores for the nonalignable-use condition. Use Blocks/Errors indicate the mean number of blocks/errors to learn both the financial advice and consultant decisions (averaged across both uses). Loan Blocks/Errors indicate the mean number of blocks/errors to learn the loan/no-loan decision.

identical to the alignable-use condition of Experiment 1. As in Experiment 1 , category use decisions were alignable between categories, in that the same dimensions predicted the same use in both superordinate categories.

Complexity was reduced in the nonalignable- use condition, relative to the alignable-u se condition, by having the participants learn only one category use decision. Half the participants in the nonalignable-use condition learned the financial advice decision, whereas the other half learned the consultant decision. Category use decisions were nonalignable between categories, in that different dimensions predicted category use within each superordinate. The design of the present experiment was such that the number of dimensions needing attention in order to learn the use of both categories remained the same between conditions.

Procedure. The procedure for the alignable-use condition was identical to that used in Experiment 1, except that the criterion for completion was extended to 16 blocks. The procedures for the participants in the nonalignable-use condition were similar to those for the participants in the alignable-use condition, with the exception that the participants learned only one of the two category use decisions. The nonalignable-use condition study criterion was no more than one mistake on a block (out of 32 total responses) or completion of 16 blocks. When the participants in either condition failed to learn the correct use(s) of the category by the 16th block, they were counted as having learned by the 17th block for analysis purposes. This was done as a conservative estimate of the next block at which they could have reached criterion had they been allowed to continue.

As in Experiment 1, we averaged the number of blocks and errors it took to learn each use in the alignable-use condition. For the participants in the nonalignable-use condition, there was only the one use (either the financial advice or the consultant decision).

\section{Results and Discussion}

The critical issue is determining whether the alignability effects exhibited in Experiment 1 resulted from betweencategory consistencies in structure predictive of category use or were due to reduced complexity in the alignableuse condition. Because the nonalignable-use condition was less complex than the alignable-use condition in the present experiment, a complexity view would predict that learning use-relevant distinctions within each category system should be facilitated, as compared with learning in the alignable-use condition. As in Experiment 1, transformations were necessary to correct for negatively skewed distributions and variability introduced by the counterbalancing manipulation. Back-transformed means and standard deviations will be reported.

Learning to make use-relevant distinctionsin a less complex, nonalignable-usecondition was not facilitated relative to that seen in a more complex, alignable-use condition. There were no significant differences between conditions with respect to either blocks to learn subclassification within both hierarchies $[t(54)<1$, n.s. $]$ or errors made during learning $\left[t(54)<1\right.$, n.s.; see Table 4]. ${ }^{7}$ Even though the participants in the alignable-use condition had to learn more than the participants in the nonalignable-usecondition, the means indicate a nonsignificant advantage for learning in the alignable-use condition on all variables.

Although it is always difficult to interpret null results, the trends in the category learning means suggest that it is unlikely that the alignability effects seen in Experiment 1 were due to reduced complexity in the alignable-use condition. In the present experiment, the participants in the alignable-use condition had to overcome a strong disadvantage in complexity, as compared with the participants in the nonalignable-use condition, yet they performed equivalently. Effect size estimates of the differences between conditions (.27 for blocks, .12 for errors; Cohen, 1988) and the superior performance in the alignable case suggest that overall complexity is not the main determinant of performance difficulty.

\section{GENERAL DISCUSSION}

Experiments 1 and 2 demonstrated the importance of between-category structural regularities in category use for the learning of multiple, use-relevant category hierarchies. Experiment 1 showed that between-category consistencies in the dimensions predictive of subclassification facilitate the learning of multiple category hierarchies. This facilitation was not due to differences between conditions in the category representations learned by participants or in the amount of information needed for subclassification in the hierarchies. Because we divorced alignability from the number of dimensions needed to use category knowledge in Experiment 1, the only difference between conditions lay in the structural regularities important for the use of the categories. When the values predicting use-relevant, subordinate level distinctions within a category were associated with the same dimensions in both hierarchies, learning was facilitated.

Experiment 2 demonstrated that the alignability effects observed in Experiment 1 could not be explained by differences between conditions in the overall complexity of the learning task. Reducing the complexity of the relations to be learned in a nonalignable category hierarchy, relative to an alignable hierarchy, did not result in facilitated learning. In fact, rather than performing better than the partic- 
ipants in the alignable-use condition of Experiment 2, the participants in the less complex, nonalignable-use condition took somewhat longer and made slightly more errors learning the category hierarchies. This is especially noteworthy given the fewer associations they were required to learn, relative to those in the alignable-use condition.

To summarize, when subordinate level distinctions within category hierarchies can be made on the basis of alignable differences between those hierarchies, learning is facilitated. This is true even when the representations and the number of dimensions needed for subclassification are held constant between conditions. Even when the overall complexity of a nonalignable condition is less than that of an alignable condition, we fail to see facilitated classification learning.

The results of Experiments 1 and 2 suggest that alignment effects on classification learning are not simply the result of differences between conditions in the objective complexity of the learning task. Rather, they reflect a commitment on the part of the learner to a type of psychological complexity-that of alignment. Part of this commitment to alignment is the belief that the structure relevant to the use of one category system will also be relevant to the use of a comparable system. In terms of learning multiple classification hierarchies simultaneously, a commitment to alignability involves the assumption that dimensions important for making use-relevant distinctions within one hierarchy will also be important for making the same types of distinctions within the other hierarchy. In the present study, the similarity of the category representations in both hierarchies (differing only in the instantiation of shared dimensions) and the uses to which they were applied probably encouraged such an assumption. When this assumption is warranted, as it is in the alignableuse condition, learning is facilitated over situations in which the assumption is not justified (as in the nonalignableuse condition).

\section{Learning Multiple Classification Systems}

Examining the learning of multiple classification systems has both provided a better understanding of how multiple categories are learned together and extended our understanding of structural alignment in category learning. More specifically, the present study suggests that the commitment to alignability is not confined to classification distinctions made within a single superordinate. It extends to whole systems of relations, such as those acquired when learning multiple, use-relevant category hierarchies.

Prior to the present study, research in which alignment effects on classification learning was examined restricted its focus to the effect of between-category consistencies and inconsistencies in the dimensions predictive of classification in categories sharing a superordinate. This research demonstrated that classification predicted by alignable differences between categories is easier to learn than classification based on nonalignable differences (Billman, 1996; Kaplan, 2000, Experiments 2 and 3; Lassaline \& Murphy, 1998; Waxman et al., 1997). Two constructs, consistent con- trast and structural alignment, have been used to explain this alignment effect in classification learning.

Consistent contrast refers to a principle of category learning in which people are biased to learn sets of categories that contrast in the same way across the categories (Billman, 1996). In such categories, the same dimensions distinguish between categories within the same contrast set (mutually exclusive categories within a superordinate). Because the principle of consistent contrast is restricted to categories sharing a superordinate, whether or not the same dimension can be used for subclassification in multiple classification systems should not affect learning (Billman, 1996). Demonstrating that the learning of multiple classification systems is affected by between-category similarities in category structures suggests that the degree to which consistent contrast explains classification learning needs to be examined further. For example, the results of the present study can be explained by consistent contrast if both the loan and the no-loan categories are considered subordinates of the superordinate category companies applying for a loan. An interesting avenue for future research would be to determine the level in a hierarchy at which categories need to share a common superordinate in order for consistent contrast to apply.

In comparison, the principle of structural alignment theory is such that it can easily be applied to predict and explain the formation of multiple classification systems. A structural alignment account of classification learning assumes that, during learning, category representations are compared in order to identify the elements of category structure important for distinguishing between category members. Part of this process involves alignment of the representations to find the maximal structurally consistent match between them (Gentner, 1983). If the representations being compared share much of their structure, the principle of structural alignment causes the learner to infer that the structure important for the use of one representation will be so for the other representation as well. This is true whether one is classifying category members into categories sharing a superordinate or learning multiple, use-relevant classification hierarchies. ${ }^{8}$

The present study demonstrated that multiple classification hierarchies are not learned independently from each other. Learning was facilitated when the structure important for subclassification within one hierarchy was also important for subclassification within the other. If a principle such as structural alignment is operating during classification learning, it can be suggested that we must extend the scope of the commitment to alignability to include whole systems of relations, such as those acquired when learning multiple, use-relevant category hierarchies.

\section{Learning the Use of Multiple Categories}

The results of the present study also inform us about the manner in which people learn to use multiple categories simultaneously. Ample evidence suggests that there is a close relationship between category use and classification. The use of category knowledge has been shown to re- 
sult in use-relevant subdivisions of domain knowledge (Boster \& Johnson, 1989; Medin et al., 1997; Ross \& Murphy, 1999). Category use has also been shown to affect classification performance (Ross, 1996, 1997, 1999, 2000).

In the category use literature, a feature prediction paradigm, such as that used in the present study, has frequently been employed to examine the effects of category use on category representation. These studies have demonstrated that the features important for satisfying category goals (use-relevant features) become more central to category representation. These features are generated more frequently in listing tasks, are judged to have occurred more frequently, and support more accurate classification than do features not important for the use of the category (Ross, 1996, 1997). Importantly, these category use effects have also been demonstrated in tasks that do not involve feature prediction (Ross, 1999). This suggests that the results of the present study might very well apply to situations in which people are required to learn the use of two categories simultaneously. If so, between-category structural regularities in the use of category knowledge will be important when learning to use multiple categories simultaneously. The implication is the same as that with classification learning: Learning to apply category knowledge is not an entirely within-category phenomenon. When learning the use of multiple categories simultaneously, we attend to between-category structural regularities in the use of category knowledge. When the same structural regularities are important for the use of similar contrasting categories, learning is facilitated over situations in which the structure important for category use differs between categories.

\section{CONCLUSION}

These experiments demonstrate the importance of between-category structural regularities in learning to make use-relevant distinctions within multiple classification systems. Because between-category consistencies in the use of category knowledge affect classification learning, it can be suggested that multiple classification systems are not learned independently of one another. The present experiments also demonstrate that the objective complexity of the learning task is not the sole explanation for the effects of such consistencies on classification learning. Rather, the results appear to reflect assumptions about the psychological complexity of the learning task. Specifically, they reflect the assumption that the category structure relevant for the use of one category system will also be relevant for the use of a similar system being learned at the same time.

\section{REFERENCES}

Anderson, J. R. (1991). The adaptive nature of human categorization. Psychological Review, 98, 409-429.

Billman, D. (1996). Structural biases in concept learning: Influences from multiple functions. In D. L. Medin (Ed.), The psychology of learning and motivation (Vol. 35, pp. 283-321). San Diego: Academic Press.
Boster, J. S., \& Johnson, J. C. (1989). Form or function: A comparison of expert and novice judgments of similarity among fish. American Anthropologist, 91, 866-889.

CoHen, J. (1988). Statistical power analysis for the behavioral sciences. Hillsdale, NJ: Erlbaum.

GENTNER, D. (1983). Structure-mapping: A theoretical framework for analogy. Cognitive Science, 7, 155-170.

Goldstone, R. (1994). The role of similarity in categorization: Providing a groundwork. Cognition, 52, 125-157.

KAPLAN, A. (2000). Alignabilityand prior knowledge in category learning. Unpublished doctoral dissertation, University of Illinois, UrbanaChampaign.

LASSALINE, M. E., \& MuRPhy, G. L. (1998). Alignment and category learning. Journal of Experimental Psychology: Learning, Memory, \& Cognition, 24, 144-160.

Medin, D. L., Lynch, E. B., Coley, J. D., \& Atran, S. (1997). Categorization and reasoning among tree experts: Do all roads lead to Rome? Cognitive Psychology, 32, 49-96.

Ross, B. H. (1996). Category representations and the effects of interacting with instances. Journal of Experimental Psychology: Learning, Memory, \& Cognition, 22, 1249-1265.

Ross, B. H. (1997). The use of categories affects classification. Journal of Memory \& Language, 37, 240-267.

Ross, B. H. (1999). Post-classification category use: The effects of learning to use categories after learning to classify. Journal of Experimental Psychology: Learning, Memory, \& Cognition, 25, 743-757.

Ross, B. H. (2000). The effects of category use on learned categories. Memory \& Cognition, 28, 51-63.

Ross, B. H., \& Murphy, G. L. (1999). Food for thought: Crossclassification and category organization in a complex real-world domain. Cognitive Psychology, 38, 495-553.

Sifonis, C. M., \& Ross, B. H. (1999, August). The effects of alignability on learning the use of categories. Poster presented at the annual meeting of the Cognitive Science Society, Vancouver, BC.

Waxman, S. R., Lynch, E. B., CAsey, K. L., \& Baer, L. (1997). Setters and samoyeds: The emergence of subordinate level categories as a basis for inductive inference in preschool-age children. Developmental Psychology, 33, 1074-1090.

WisNieWski, E. J., \& MARKMAN, A. B. (1997). Feature alignment in concept learning. Unpublished manuscript.

YAMAUCHI, T., \& MARKMAN, A. B. (2000).Learning categories composed of varying instances: The effect of classification, inference, and structural alignment. Memory \& Cognition, 28, 64-78.

\section{NOTES}

1. It is important to state that even though we are studying the formation of use-relevant category hierarchies, we are not making any claims as to the manner in which use-relevant category hierarchies are learned, as compared with taxonomic hierarchies. Examining the role of contrastive information in learning use-relevant hierarchies will extend our knowledge of the subject. Whether or not taxonomic category hierarchies are learned in the same fashion will not be addressed in the present paper.

2. Even though Sifonis and Ross's (1999) study was designed to examine category use learning, their participants could be said to be learning multiple classification hierarchies. Deciding which of two types of financial advice to provide an applicant can be viewed as a classification task in which the loan/no-loan categorization is further divided into one of two subcategories (Anderson, 1991). For each category, one subcategory receives one type of financial advice, with the other receiving the other type of advice. Even though there is some evidence suggesting that the manner in which a task is presented (e.g., as either classification or feature prediction) affects task performance (Yamauchi \& Markman, 2000), it is possible for participants to view the prediction as a subclassification. To facilitate discussion of the issues in the present study, we will continue to refer to Sifonis and Ross's study as one examining subclassification, rather than category use.

3. The focus of the present study is on the effects of alignability in the dimensions predictive of subordinate level distinctions on the learning of 
multiple classif ication systems. Although we will report the means for classification learning, we will not discuss alignability effects on learning these distinctions in the present paper. We will present statistics in the notes, for interested readers.

4. There were no significant alignability effects on learning the superordinate level distinctions in either the number of blocks needed to learn the classification $[t(46)=1.40$, n.s. $]$ or the number of errors made during learning $[t(46)=0.86$, n.s. $]$.

5 . We would like to thank an anonymous reviewer for this explanation of the results.

6. The participants for Experiment 2 were drawn from a different pool than those from Experiment 1. Pretesting indicated that the number of blocks to reach criterion would have to be increased to allow the participants from this pool time enough to learn the classification hierarchies.
7. There were no significant alignability effects on learning the superordinate level distinctions in either the number of blocks needed to learn the classification $[t(54)=0.89$, n.s. $]$ or the number of errors made during learning $[t(54)=0.86$, n.s. $]$.

8. Structural alignment theory does not specifically predict whether or not multiple classification systems will be learned independently. However, if comparisons are made between systems during learning, structural alignment theory allows for structural consistencies and inconsistencies between systems to affect learning.

(Manuscript received September 30, 1999; revision accepted for publication July 11,2002.) 